\title{
The quasar clustering and its evolution in a semi-analytic model based on ultra high-resolution $N$-body simulations
}

\author{
Taira Oogi ${ }^{1}$, Motohiro Enoki ${ }^{2}$, Tomoaki Ishiyama ${ }^{3}$, Masakazu A. R. \\ Kobayashi $^{4}$, Ryu Makiya ${ }^{5}$ and Masahiro Nagashima ${ }^{1}$ \\ ${ }^{1}$ Faculty of Education, Bunkyo University, 3337 Minami-Ogishima, Koshigaya-shi, \\ Saitama 343-8511, Japan \\ email: oogi@koshigaya.bunkyo.ac.jp \\ ${ }^{2}$ Faculty of Business Administration, Tokyo Keizai University, Japan \\ ${ }^{3}$ Institute of Management and Infomation Technologies, Chiba University, Japan \\ ${ }^{4}$ Research Center for Space and Cosmic Evolution, Ehime University, Japan \\ ${ }^{5}$ Institute of Astronomy, the University of Tokyo, Japan
}

\begin{abstract}
We investigate clustering properties of quasars using a new version of our semianalytic model of galaxy and quasar formation with state-of-the-art cosmological $N$-body simulations (Ishiyama et al. 2015; Oogi et al. 2015). We assume that a major merger of galaxies triggers quasar activity. We find that the quasar bias does not depend significantly on the quasar luminosity, similar to observed trends. This result reflects the fact that quasars with a fixed luminosity have various Eddington ratios and thus have various host halo masses that primarily determine the quasar bias. The quasar bias increases with redshift, which is in qualitative agreement with observations. Our bias value is lower than the observed values at high redshifts, implying that we need some mechanisms that make quasars inactive in low-mass haloes and/or that make them more active in high-mass haloes.
\end{abstract}

Keywords. galaxies: formation, quasars: general, large-scale structure of universe

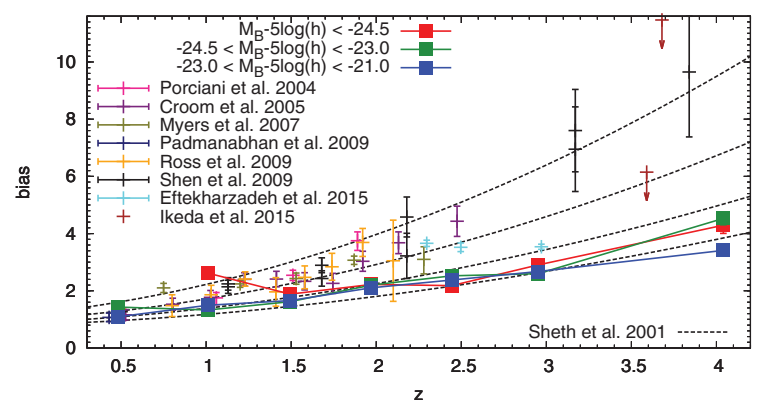

Figure 1. Redshift evolution of the bias of bright, intermediate and faint quasars in our model (red, green and blue filled squares). The dashed lines are halo bias factor evolution for fixed halo mass of $\log \left[M_{\text {halo }} /\left(h^{-1} M_{\odot}\right)\right]=11.5,12.0,12.5$ and 13.0 from bottom to top, respectively, using the Sheth et al. (2001) fitting formula with the Planck cosmology. Observational results are also plotted (plus signs and error bars).

\section{References}

Ishiyama, T., Enoki, M., Kobayashi, M. A. R., Makiya, R., Nagashima, M., \& Oogi, T. 2015, PASJ, 67, 61

Oogi, T., Enoki, M., Ishiyama, T., Kobayashi, M. A. R., Makiya, R., \& Nagashima, M. 2015, $M N R A S$ (Letters), accepted

Sheth, R. K., Mo, H. J., \& Tormen, G. 2001, MNRAS, 323, 1 